\title{
Inhaltsverzeichnis Jahrgang 1910
}

\section{Abhandlungen und Notizen.}

Barth, J., Bemerkungen zu mischnischem הָָָָ Bolssier, A., A propos des Nephilîm . . . Čajkanovið, V., Ein punisches Sprichwort bei Augustin

Dittrich, ., Platons Zablenrätsel u. die Präzession. Mit 3 Abbildungen

Frank, O., Zum asgyrischen Lexikon . . .

Fries, K., Zur Ilias . . . . . . . . . . 473

-, Sư̌anna

Goldziher, J., Tumfum al Hindī .

Grimme, H., Midian . . . . . . . .

Herrmann, J., Die Zahl zweiundvierzig im AT

Holma, H., Zum assyrischen Wörterbuch . .

Forten, M., Der Gang nach dem Eisenhammer

Jirku, A., לחי ראי .

Lansdon, St. Concerning the Element $l a$ in Babylonian Names

-, The sign Thy

Marmorstein, $\triangle$., Einige hebräische Redensarten

Meisener, B., Bemerkangen zu CT XXV .

Müller, W. M., Die altägyptischen Namen für Kreta und Zypern

- Bemerkungen zu den neuesten Funden Petries

_, Die Königsbinde der abessynischen Herrscher

Poiger, F. E., Zur Agum-Inschrift

- Zur altbabylonischen Datierungsweise II. Mit 1 Abbildung

-, Zu meiner Glossentheorio

Paebel, A., Das Datum des 24. Jahres Samsuilunas

Seyoe, A. H., Tid'al and Tantalos

Sohmitz, M., Herkunft des Namens Marokko

Schnabel, P., Apokalyptische Berechnung der Endzeiten nach Berossos

-, Der erste König der IV. Dynastie der Liste A

Schollmeyer, A., Ippirn = Kummer . . . .

Sohultz, W., Simsons Rätsel

-, Das Flies des Gideon

Strause, O., Noch einmal die Zahl 42 im AT .

Thureau-Dangin, Fr., Le premier roi de la quatrième dynastie babylonienne . . . . .

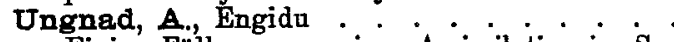

- Einige Fälle progressiver Assimilation im Sumerischen

—, Zor Schaltungspraris in der Hammurapi-Zeit

Weber, O., Zu Eislers Notiz OL 1909 Sp. $482 \mathrm{f}$. (Grabschrift in Petra betr.)

Wiedemann, A., Zur Kefti-Frage

Winokler, H., Die Arier in den Urkunden von Boghaz-köi

- Ein nenes Prisma Sinacheribs im British Maseum . . . . . . . . . . . .

Wreszingli, $\dot{W}$., Zar agyptisehen Kultur- und Kunstgeschichte. Mit 1 Tafel . . . . . .

-, Die Inschriften des Monthembet im Tempel der Mut. Mit 4 Tafeln . . . . . . . .

\section{Besprechungen.}

Aptowitzer, V.: Die syrischen Rechtsbücher. Bespr. v. F. Holldack de la sàde et de

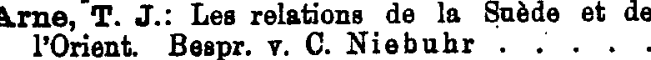

400

306

493
Aufreoht, Th.: Die Sanscrit-Handschriften der

* K. Hof- und Staatsbibliothek in München. Bespr.

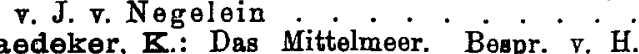
Baedeker, K.: Das Mittelmeer. Beapr. v. H Barton, G. A.: Documents from the Temple Archives of Telloh. Bespr. .. A. T. Olay . .

Baver, L.: Das Pallistinensische Arabisch. Bespr. v. M. Löhr

van Berohem, M., et M. Sobernheim: Matériaux pour un Corpus Inscriptionum Arabicarum II, 1. Bespr. v. E. Mittwoch. . . . . .

Berriedale Keith, A.: Aitareya Āranyaka. Bespr. v. J. v. Negelein

Bertholet, A.: Das Linde des jüdischen Staatswesens. Bespr. v. J. Herrmann . . . .

von Bissing, B.: Les débuts de la statuaire en Egypte. Bespr. v. W. Wreszinski . . .

Blau, L.: Bacher Vilmos élete és mükơdése. Bespr.

v. F. Perles .
Böklen, E.: Sneewittchenstadien I. Bespr. W. Schultz . · $\dot{\text { Geschichte Aegyptens. Deutsch }}$

Breasted, J. H.: Geschichte Aegyptens. Deutsch von H. Ranke. Bespr. จ. A. Wiedemann 12

Religion und Sitte. Bespr. v. F. N. Finck.

Brockelmann, C.: Katalog der orientalischen Handechriften der Stadtbibliothek zu Hamburg I. Bespr. v. M. Hartmann . . . . . .

Catalogue of the Arabic and Persian Mss in the Oriental Public Library at Bankipore. Bespr. v. J. Horovitz

Catalogue des Monuments et Inscriptions de l'Egypte Antique, Tome III. Kom Ombos. 20 partie, fasc. 3. Bespr. v. W. M. Miller.

Ohauvin, V.: Bibliographie des ouvrages arabes ou relatifs aux Arabes publiés dans l'Europe chrétienne de 1810-1885. Vol. XI. Bespr. ₹. Tr. Mann . The Religion of Ancient Palestine.

Cook, St. A.: The Religion of Ancient Palestine. Bespr. v. J. Herrmann ${ }^{-}$. Religionen im

Spalte

461

498

131

504 römischen Beidentum. Deutsch ron C. Gebrich. Bespr. v. C. Fries'

Dalman, G.: Palästinajabrbuch des Deutschen evangelischen Instituts für Altertumskunde des hl. Landes za Jerusalem V. Bespr. v. J. Herr$\operatorname{mann}$.

Delitzsch, Fr.: Handel und Wandel in Altbabylonien. Bespr. v. Mario Pancritius. . - Asurbanipal und die assyrische Kultur seiner Zeit. Bespr. v. M. Pancriting . . . .

Dleterioh, A.: Eine Mithrasliturgie. 2. Aufl.

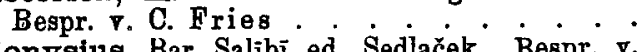

Dionysius Bar Șalibì ed. Sedlaček. Bespr. v. E. Nestle dio ägyptische Religion. 2. Aufl. Beepr. v. A. Wiedemann .. . . . . .

Falls, J. O. F.: Siwah. Die Oase des Sonnengottes in der libyschen Wüste. Bespr. v: W. M. $M$ üllor

Fimmen D.: Zeit und Dauer der kretiech-my-kenischen Kultur. Bespr. v. W. M. Müller Frankfurter, S.: Das altjüdische Erziehungs- und Unterrichtswesen. "Bespr. v. H. Vogelstein 
Freund, L.: Zur Geschichte des Ehegüterrechts. Bespr. v. F. Holldack. . . . . . • .

Friedlander, J.: Selections from the Arabic Writings of Maimonides. Bespr. v. M. Horten

Galtier, E.: Foutouh al Bahnast. Bespr. v. H. Reckendorf

Gardiner, A. H.: Die Erzählung des Sinuhe und die Hirtengeschichte. Bespr. v. W. M. Müll or

Gauthier, L.: Ibn Thofail, sa vie, ses ceuvres. Bespr. v. M. Horten

-, La theorie d'Tbn Rochd. Bespr. v. M. Horten

Gautier, M. J. E.: Archives d'une famille de

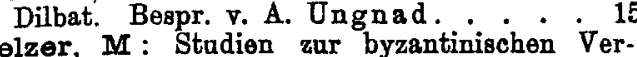
Gelzer, M : Studien zur byzantinischen Ver-
waltung Aegyptens. Bespr.. . W. Wiedemann

Ginzberg, L.: Geonica I, II und

-, Yerushalmi Fragments I. Bespr. v. F. Perles

-, The Legends of the Jews. Translated by $H$. Szold I. Bespr. V. F. Perles

Graetz, H.: Geschichte der Juden Bd. V. Bespr. v. W. Bacher.

Grothe, B.: Meine Studienreise durch Vorderasien und

-, Geographische Charakterbilder aus der asiatischen Türkei und

-, Wanderungen in Persien. Bespr. v. F. Hommel

จ. Hahn, O.: Erster Versuch oiner Erklärung kaukasischer geographischer Namen. Bespr. v. F. Bork

Hahn, \#.: Die Entstehung der Pflagkultur. Bespr. v. E. Brandenburg. Mit 1 Abbildung

Hertmann, M. Der Islamische Orient. Berichto und Forschungen Bd.III. Bespr. v. H. Winckler

Hell, J.: Die Kultur der Araber. Bespr. v. H. Reckendorf

Herzog, Fr. A.: Die Träger der Offenbarung im Rahmen der Weltgeschichte. Bespr. v. S Landersdorfer.

Heuzey, L., et Fr. Thureau-Dangin: Restitution mat́rielle de la Stèle des Vautours. Bespr. v. F. E. Poiser. . . . . . . . .

Houtsma, M. Th., und A. Sohaade: Enzyklop\&die des Islam Lief. 1. Bespr. $\nabla . J$. H o r o vitz

Hüising, G.: Die iranische Ueberlieferang und das arische System. Bespr. ₹. E. Sieck $\theta$.

Jastrow, M. jr.: Die Religion Babyloniens und Assyriens. Bespr. v. M. Pancritins i 199.

Ibn Saad: Biographien Muhammeds usw. Bd.IV, 2 hrsg. v. J. Lippert, n. Bd. VI, hrsg. v. K. V. Zetterstéen. Bespr. v. H. Reckend orf

Jeremias, A.: Das Alter der babylonischen Astronomie. Bespr. \%, W. Erbt

Iselin, I. F.: Der morgenlandische Ursprung der Grallegende. Bespr. v. W. Weyh . .

Kahle, P.: Zur Geschichte des arabischen Schattentheaters. Bespr. v. J. Horovitz . . . . Berichtigung (J. Horovitz)

Kiepert, R.: Karte von Kleinasien, Blatt $\dot{D} 4$, Adana. Bespr. v. E. Herzfeld

Klein, S.: Beiträge zar Geographie und Geschichte Galilias. Bespr. v. A. Marx . . . . .

Kohl, H., Kasr Firaun in Petra. Bespr. v. E. Herzfeld

Kortleitner, F. A.: De Hebraeorum ante exsilium Babylonium monotheismo. Bespr. v. F. Heh'n

Kropat, A.: Die Syntax des Antors der Chronik verglichen mit der seiner Quellen. Bespr. v. A. Ungnad .

Landberg, Oarlo Graf von: Jeder tat, was ihm passt. Bespr. v. H. Reckendorf.

Landersdorfer, s.: Altbabylonische Privatbriefe. Bespr. Ү. H. Pick : . . . . . . . .
Spalte

Leder, H.: Das geheimnisvolle Tibet. Bespr. v. A. Grünwedel . . . . . . . . . .

v. Lichtenberg, R. Frh.: Haus, Dorf, Stadt. Bespr. ఛ. E. Herzfeld .

Margoliouth, J. P.: Extracts from the Ecclesiastical History of John of Ephesus. Bespr. V. Eb. Nestle.

69 Martin, Fr.: Lettres Néo-Babyloniennes. Bespr. Y. S. Landergdorfer

441 Maspero, G.: Au temps de Ramsès et d'Assourbanipal. Bespr. v. W. Wreszinski . .

Meinhold, H.: Sabbat und Sonntag. Bespr. v. J. Herrmann . . . . . . . . .

Merx, A.: Der Messias oder Ta'eb der Samaritaner. Bespr. v. W. Bacher . . .". .

Meyers Reisebuicher. Das Mittelmeer und seine Küstenstädte, Madeira und Kanarische Inseln. Bespr. .. F. B ork . . . . . . . . .

Möller, G.; Hieratische Lesestücke für den akademischen Gebrauch, Heft2. Bespr. v. H. Ranke

77 -, Hieratische Palaographio Bd. II. Bespr. v. H. Ranke

de Morgan, J.: Les premières Civilisations. Bespr. จ. E. Brandenburg . . . . . . . .

Myhrman, M.: Kitāb mưìd an-nï am wa-mubìd an-niqam. Bespr. $\nabla$. H. Reckendorf .

Nicholson, R. A.: Elementary Arabic. Second Rearding Book. Bespr. i p. v. F. Bork.

412 Nikel, J., und J. Rohr: Biblische Zeitfragen. Erste Folge Heft 1-4. Zweite Folge Heft 7. Bespr. v. S. Landersdorfer. . . . . .

Noldeke, A.: Das Heiligtum al-Ḥusains in Kerbela Bespr. v. E. Herzfeld .

Noldeke, Th., u. Fr. Sohwally: Geschichte des Qorāns I. 2. Aufl. Bespr. v. H. Grimme

Oldenberg, H.: Rgreda, Textkritische and exegetische Noten. Buch I-VI. Bespr. v. J. ₹. Negelein

Partach, J.: Des Aristoteles Buch "Ueber das Steigen des Nilg*. Bespr. จ. E. Herzfeld

197 Peters, N.: Die jüdische Gemeinde von Elophantine-Syene. Bespr. v. W. Staerk . .

31 peckham, G. $\mathbf{A}$.: An Introduction to the study of Obadjah. Bespr. v. J. Herrmann.

165 Poebel, A.: Babylonian Legal and Business Documents. Bespr. v. A. Ungnad . Puukko, J.: Das Deuteronomium. Bespr. v. W. Staerk

Ravn, O. H.: Om nominernes bqjning i Babylonisk-Assyrisk. Bespr. v. K. Tallqvist.

von Reitrenstein, F. Frh.: Liebe und Ehe im alten Orient. Bespr. v. C. Fries. . . .

Sarre, Fr.: Erzengnisse islamischer Kunst. Bespr. v. E. Herzfeld . . . . . . . . . .

Soheil, V.: Annales de Tukalti-Ninip ח. Bespr. v. H. Winckler.

v. Schlichterroll, C. F.: Liebesleben im klassichen Altertam. Bespr. 7. C. Fries .

648 Schriften des Vereins Mekize Nirdamim, 3. F. 1-4. Bespr. v. F. Porles . . . . .

501 Sethe, K.: Die Einsetzung des Veziers unter der

413 18. Dynastie. Bespr. v. W. M. Muller : bole. Bespr. v. H. W inckler. Mit 1 Abbildung 17. 68

311 Sources Syriaquea I Mšiha-zkha. Bar Penkaje.

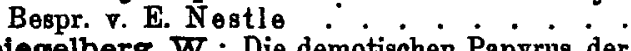

Spiegelbere, W.: Die demotischen Papjrus der Musées royaux du Cinquantenaire. Bespr. v.

W. M. Muller . . . . . . . . . . . 212

127 จ. Spruner: Historischer Handatias I, Lief. 6. Bespr. T. E. Herzfeld

23 Stahn, E.: Die Simmonsage. Bespr. v. W.Schultz
Spalte

321

406

260

266

185

307

272

224

79

449

217

80

414

542

370

115

499

114

185

112

186

172

540

277

442 
Steindorff, G.: Die ägyptischen Gaue und ihre politische Entwicklung. Bespr. v. A. Wied o$\operatorname{mann} \cdot \cdot \cdot \cdot \cdot \cdot \cdot \cdot \cdot \cdot \cdot \cdot \cdot \cdot$

Struok, A.: Mistra, eine mittelalterliche Ruinenstadt. Bespr. v. E. Brandenburg van Tiohelen, Th.: Land en Volk in Palestina. Bespr. v. F. B ork

Torge, P.: Seelenglaube und Unsterblichkeitshoffnung im Alten Testament. Bespr. v. K. Fries

Triandaphyllidis, M. $\dot{A}:$ Die Lehnworter in der mittelgriechischen Volgärliteratur. Bespr. v. F. Bork .

Venetianer, L.: Ursprang und Bedeutung der Prophetenlektionen. Bespr. v. H. Vogel stein

Viollet, M. H.: Description du palais de alMoutasim, fils d'Haroun-al-Raschid à Samara. Bespr. v. J. Strzy gow aki

Virolleaud, Oh.: L'Ástrologie Chaldéenne. Bespr. v. A. Boissier . . . . B

Weill, R.: Le séjour des Isrálites au désert et le Sinai dana la relation primitive. Bespr. v. A. Wiedemann. .

Windisoh, H.: Der messianische Krieg und das Urchristentum. Bespr. v. W. Soltau . .

Winkler, Heinrich: Der ural-altaische Sprachstamm, das Finnische und das Japanische. Bespr. r. R. Fuchs. .

Wressingki, W.: Der grosse medizinische ${ }^{\circ}$ ' pyrus des Berliner Museums. Bespr. $\nabla$. H. Ranke •. . . . . . . .

Wünsche, $\mathbf{A}$ : Aus Israels Lehrhallen IV். Bespr. จ. F. Perles

Zeitlin, M.: Le style administratif chez les Assyriens. Bespr. $\nabla$. S. Landersdorfer

Zimmern, H.: Der babylonische Gott Tamūz. Bespr. v. M. Pancritius. . . . . . .

\section{Verzeichnis der Rezensenten.}

Bacher, W. . 77. 315

Boissier, A. . 73

Bork, F. 185. 224. 412.

$$
461.546
$$

Brandenburg, E. 119.

Clay, A. T. . . . 264

Erbt, $\dot{W} . \vdots \vdots 545$

Finck, F. N. $: \begin{array}{r}33 \\ \hline\end{array}$

Fries, C. 124. 185. 224. 411

Fuchs, R. . . . 456

Grimme, H. 217. 225

Grünwedel, $\dot{A}$. . 371

Hartmann, M. . 503

Hehn, F. . . . 311

Herrmann, J. 26. 213. 266. 370.500

Herzfeld, E. 214. 277. 321. 413. 414. 449 . 548 Holldack, F. . . 175

Hommel, F. . . 549

Horovitz, J. 31. 128.

130. 279

Horten, M. 90. 441. 539

Landeredorfer, $S$. 79 .

82. 260.403

$\mathrm{L} 8 \mathrm{hr}, \mathrm{M} . \cdots .498$

Mann, Tr. 219

Marx, A. . : 501

Mittroch, E.: 131
Spalte

Sprechsaal.

Fischer, A.: Das Mannesmannsche Berggesetz Ginzberg, L.: Mechiltha (Aus einem Briefe an F. Perles). . . . . . . . . . . .

454 Grimme, H.: Rothsteins Randbemerkungen zu meiner Besprechung seiner „Grundzüge ${ }^{4}$. . 83

546 Ka mpffmey er, G.: Das sogenannte marokkanische Berggesetz

Kugler, F. X: Erwiderung auf Dr. Dittrichs Platons Zahlenrätıel und die Präzession . . . 277

Perles, F.: מדין = südarabisch מר̄ . . . . . 134

461 Weissbach, F. H.: Znr altporsischen Keilschrift 414

27

320

73

Kgl. Museen Berlin 86. Kloinasien . 510

288. 510. 555 Kreta. . . 510. 555

Messtellung mohamme-

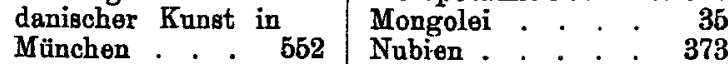

174

Aegypten 34. 186. 323

Griechenland . . 34. 556 Palästina : . : : 86

Italien. . . . . 228 Turkestan :. : 34

Aus gelehrten Gesellschaften.

456 Académie des Inscriptions, Paris 35. 87. 88, 186. 325.

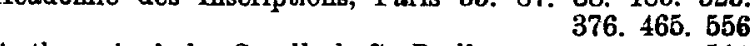

Anthropologische Gesellschaft, Berlin -... . . 611

Königl. Săchs. Gesellschaft der Wissensch., Leipzig 325

161 Vorderasiatisehe Gesellschaft, Berlin . . . . . 325

403

Vereinigung für ästhetische Forschung 35. - Geplante Oesterreichische Expedition nach Aegypten 35. - Tieropfer in russisch-persisch-türkischen Grenzgebieten 35. - Ausstelingy mohammedanischer Kunst in München 35. 552. - Pierpont Morgan Stiftung 88. - College von Aligarh 134. - Hohlenstäd te im Hinterlande von Tripoli 186. - Wissensehaftliche Bibliothek in Tanger 326. - Expedition der "Jewish territorial Organisation“ 326. - Mission de Gironcourt im Sndan 376. - Manernkranz ron Byzanz in Konstantinopel 376. - Albert Gayet's Ergebnisse seiner Kampagne 465. - Vermüchtnis Josef Flügel 511. - Aelteste papierne Urkunde in griechischer und arabischer Sprache 511. - Studeriten der Theologie in Athen 511. - Italienische archäologische Schule in Athen 511. - Ausgrabung von Tel Halaf 557. - Islamkundo im Seminar für orientalisch. Sprachen, Berlin 557. - Ausgrabungen der Unir. Oxford in Faras 657 .

Pick, H. ' ' Reckendorf, H. 127. 222 223. 272. 407

Schultz, W. 442. 504 Siecke, E. . . . 165 Soltau, W. - . 268 Staerk, W. . 499. 542 Strzygorski, J. . 320 Tallqvist, $\mathrm{K}$. 114 Ung nad, A. 115. 156. 204.

Vogelstein, H. 27. 438 Woyh, W . . . 359 Wiedemann, A. 122. 174 . 210. 275. 310. 647

Winckler, H. 17. 68. 112.

Wroszinski, W. 437.648

\section{Personalien.}

Beer, G. 36. - Bénédite, I. 512. - Brockelmann, K. 90. - Dussaud, R. 512. - Euringer, P. 466. - Finck, F. N. 228. - Foncart, H. 657. - Frank, C. 90. Hamdy Bey 135. - Haynes, J. H. 465. - Jacob, G. 466. - Jequier, G. 557. - Krumbacher, K. 36. - Ledrain 135. - Lowy, E. 376. - Macalister, R. A. St. 512. - Michon, E. 512. - Niese, B. 187. - de Nolhac, P. 512. - Pottier, E. 512 . - Rassam, Horm. 511. Schmidt, C. 88. - Schulthøse, F. 228. - Suhürer, E. 228. - de Vogu6, M. 326. - Ziegler, K. 90.

Druckfehterberichtigung: 238.415.

Briefkasten: 279.

Zeitschriftenschau: Am Schlusse jeder Nummer. 